\title{
Inoculação de fungos micorrízicos arbusculares e adubação fosfatada em mudas de cajueiro-anão-precoce
}

\author{
Olmar Baller Weber ${ }^{(1)}$, Carmem Cristina Maréco de Souza ${ }^{2}$, \\ Darcy Mayra Furtado Gondin(3), Francisco Nelseudes Sombra Oliveira(1), \\ Lindbergue Araújo Crisóstomo ${ }^{(1)}$, Ana Lucy Caproni( ${ }^{(4)}$ e Orivaldo Saggin Júnior(5)
}

\begin{abstract}
(1)Embrapa Agroindústria Tropical, Rua Dra. Sara Mesquita, 2270, Planalto Pici, CEP 60511-110 Fortaleza, CE. E-mail: weber@cnpat.embrapa.br, sombra@cnpat.embrapa.br, linberg@cnpat.embrapa.br (2)Universidade Federal do Ceará, Av. Mister Hull s/no, CEP $60021-970$ Fortaleza, CE. (3)Universidade Estadual do Ceará, Av. Parajana, 1700, CEP 60740-000 Fortaleza, CE. (4)Fundação Mokiti Okada/Embrapa Agrobiologia, Caixa Postal 74505, CEP 23851-970 Seropédica, RJ. E-mail: lucycaproni@bol.com.br ${ }^{(5)}$ Embrapa Agrobiologia. E-mail: saggin@cnpab.embrapa.br
\end{abstract}

\begin{abstract}
Resumo - O objetivo deste trabalho foi avaliar os efeitos da inoculação de fungos micorrízicos arbusculares (FMA) nativos e exóticos e da adubação fosfatada no crescimento e no acúmulo de nutrientes em mudas do cajueiro-anão-precoce CCP 76. O experimento, realizado em casa de vegetação, constou de um arranjo fatorial (4x2), sendo três misturas (duas comunidades de FMA nativos e o produto comercial Mycogold) mais o controle sem fungo e duas doses de fósforo ( 0 e $87 \mathrm{mg} / \mathrm{L}$ ). Os fungos nativos eram formados pelas espécies Glomus etunicatum, G. glomerulatum, Scutellospora sp. e Acaulospora foveata, da primeira comunidade, e G. etunicatum, Entrophospora sp. e Scutellospora sp., da segunda comunidade. Observou-se resposta das mudas do cajueiro ao fósforo aplicado no solo. A associação simbiótica com os fungos da primeira comunidade e do produto comercial foi vantajosa no desenvolvimento das mudas do cajueiro, aos quatro meses da semeadura.
\end{abstract}

Termos para indexação: Anacardium occidentale, Glomus, fósforo, absorção de nutrientes, propagação do caju.

\section{Inoculation of arbuscular micorrhizal fungi and phosphate fertilization on dwarf cashew seedlings}

\begin{abstract}
This work aimed to evaluate the effects of the inoculation of native and exotic arbuscular micorrhizal fungi (AMF) and of the phosphate fertilization on plant growth and nutrient accumulation in dwarf cashew CCP 76. A greenhouse experiment, using a factorial arrangement ( $4 \times 2$ ), with three mixtures (two native communities of AMF and the commercial Mycogold product) besides a control without fungi and two dosages of phosphorus ( 0 and $87 \mathrm{mg} / \mathrm{L}$ ) was carried out. Native AMF were represented by the species Glomus etunicatum, $G$. glomerulatum, Scutellospora sp. and Acaulospora foveata, from the first community, and G. etunicatum, Entrophospora sp. and Scutellospora sp., from the second community. The cashew seedlings presented a low response to the phosphorus treatment. The symbiotic plant association with the native fungi of the first community and exotic fungi of the commercial product allowed a better plant growth four months after cashew nut sown.
\end{abstract}

Index terms: Anacardium occidentale, Glomus, phosphorus, nutrient uptake, cashew propagation.

\section{Introdução}

A cultura do cajueiro destaca-se na Região Nordeste do Brasil, com extensas áreas exploradas, principalmente nos Estados do Ceará (356 mil ha), Piauí (147 mil ha), Rio Grande do Norte (116 mil ha) e Maranhão (12 mil ha), gerando uma produção anual superior a 186 mil toneladas de castanhas (Agrianual, 2002). Esses dados indicam a baixa produtividade da cultura $(295 \mathrm{~kg} / \mathrm{ha}$ de castanhas), o que impede ganhos satisfatórios com o sistema de produção (Barros et al., 2000).

Vários são os fatores que afetam a produção do cajueiro, como a variabilidade genética das plantas (Barros et al., 2000), o uso da água de irrigação (Almeida et al., 2002; Oliveira et al., 2003) e o manejo do solo (Oliveira \& Ramos, 1995). As principais áreas com cajueiro localizam-se na faixa litorânea do Nordeste, onde prevalecem os solos de Tabuleiros Costeiros que são 
bastante arenosos e apresentam baixa fertilidade natural (Oliveira \& Ramos, 1995).

As mudas do cajueiro são obtidas tradicionalmente das castanhas, em sacolas ou em tubetes de plástico, contendo misturas de substratos orgânicos e solo (Chaves et al., 2000), entre outros materiais inertes (Souza, 2001). Os substratos orgânicos dos viveiros devem ser previamente estabilizados, mediante o processo de compostagem, e o solo desinfestado, para evitar a contaminação por patógenos do solo. Ghini (1997) demonstrou que a solarização do solo foi eficiente para a eliminação de patógenos. Contudo, os tratamentos de desinfestação reduzem ou eliminam microrganismos benéficos para as plantas, como os fungos micorrízicos arbusculares (FMA), propiciando a formação de mudas isentas da simbiose micorrízica arbuscular ou associadas a espécies de FMA pouco eficientes.

Em condições naturais, o cajueiro forma associação com FMA (John, 1980; Chulan et al., 1983; Haugen \& Smith, 1993). Contudo, os FMA variam em eficiência na promoção do crescimento das plantas, sendo desejada a seleção de espécies mais efetivas. Haugen \& Smith (1993) aplicaram em viveiro comercial da Austrália a mistura comercial NutriLink, contendo esporos de Glomus intraradices, e não observaram incremento no crescimento das mudas do cajueiro. Cardoso (1994) também não observou resposta no crescimento das plantas, aos 60 dias de idade, ao aplicar suspensões com esporos das espécies Glomus sinuosum, Glomus macrocarpum, Glomus versiforme, Glomus sp., Scutelospora heterogama e Scutelospora sp. nas raízes das plântulas do cajueiro-anão-precoce CCP 06 cultivado em vasos.

A ineficiência de FMA no período inicial de crescimento das mudas poderia estar relacionada à baixa capacidade dos fungos em colonizar as raízes, à utilização das reservas de nutrientes pelas plantas e à alta capacidade das plantas em absorver nutrientes do solo, principalmente o fósforo. Até 75 dias de idade, no viveiro, Haugen \& Smith (1993), Cardoso (1994) e Lima et al. (2001) observaram ausência de resposta do cajueiro à aplicação de P no solo. Porém, Lima et al. (2001) verificaram melhor crescimento das mudas em substrato acrescido de coprólito de minhoca.

O manejo de FMA seria facilitado no viveiro pela mistura de inóculos ao substrato, o que poderia resultar em mudas mais vigorosas e mais resistentes aos estresses ambientais. Segundo Siqueira \& Franco (1988) e Sieverding (1991), as plantas micorrizadas apresentam maior vigor e são mais tolerantes aos fatores adversos do solo e do ambiente e ao estresse com o transplante para o campo. $\mathrm{Na}$ implantação de pomares com cajueiro é comum perdas bem superiores a $10 \%$ das mudas transplantadas, o que eleva o custo de produção.

Acredita-se no benefício da micorrização por períodos mais prolongados no viveiro, bem como no estabelecimento e no desempenho das mudas de cajueiro no campo. Na formação do pomar, o cajueiro responde à adubação mineral, sendo aplicados na cultura, $50 \mathrm{~g} /$ planta de $\mathrm{P}_{2} \mathrm{O}_{5}$ e $40 \mathrm{~g} /$ planta de $\mathrm{K}_{2} \mathrm{O}$ (Oliveira et al., 2000).

$\mathrm{O}$ objetivo deste trabalho foi avaliar os efeitos da inoculação de FMA nativos e exóticos e da adubação fosfatada no crescimento e no acúmulo de nutrientes em mudas do cajueiro-anão-precoce CCP 76.

\section{Material e Métodos}

O experimento com mudas do cajueiro-anão-precoce (Anacardium occidentale, L.), clone CCP 76, foi realizado em vasos na casa de vegetação da Embrapa Agroindústria Tropical, de fevereiro a junho de 2002. Os tratamentos constituíram um arranjo fatorial $4 \times 2$, sendo três misturas (duas comunidades de FMA nativos e o produto comercial Mycogold, contendo fungos do gênero Glomus) mais o controle sem fungos e duas doses de $\mathrm{P}$ ( 0 e $87 \mathrm{mg}$ de $\mathrm{P}$ por litro de solo), distribuídos no delineamento inteiramente casualizado, com cinco repetições. A fonte de fósforo foi $\mathrm{K}_{2} \mathrm{HPO}_{4}$. O produto comercial era proveniente da University Kebangsaan Malaysia, Selangor, Malásia.

Os fungos nativos foram isolados de raízes finas (diâmetro de aproximadamente $1 \mathrm{~mm}$ ) do cajueiro-anãoprecoce, provenientes de pomares dos Municípios de Horizonte, constituindo a comunidade formada pelos fungos Glomus etunicatum, G. glomerulatum, Scutellospora sp. e Acaulospora foveata (CA1) e de Pacajús, constituindo a comunidade formada pelos fungos G. etunicatum, Entrophospora sp. e Scutellospora sp. (CA2), do Estado do Ceará. Os propágulos de FMA associados às raízes do cajueiro foram multiplicados em plantas de sorgo, em vasos contendo a mistura autoclavada de areia e vermiculita, na proporção 1:1. Após dois meses de cultivo do sorgo, os esporos de FMA prevalentes na rizosfera das plantas foram separados, e novamente multiplicados com a mesma planta hospedeira, para obter-se os inóculos de FMA nativos. Estes, sendo constituídos da mistura de solo e areia, 
fragmentos de raízes de sorgo, esporos e micélio fúngico, foram secados à temperatura ambiente e guardados com a finalidade de estabelecer os tratamentos e identificarem-se as espécies fúngicas presentes nas misturas. $\mathrm{Na}$ identificação dos fungos nativos foram extraídos os esporos do solo, pela técnica do peneiramento úmido, e estes montados sobre lâminas e cobertos com álcool polivinílico a 75\% (Sieverding, 1991) e ácido lático, para observar suas características morfológicas ao microscópio (100 a 1000x), conforme Shenck \& Perez (1988).

O solo utilizado no experimento foi coletado da camada arável $(0-20 \mathrm{~cm})$ de um Argissolo ocupado por cajueiros, no campo experimental da Embrapa Agroindústria Tropical, em Pacajús, CE. O solo livre de raízes foi autoclavado a $120^{\circ} \mathrm{C}$ por 30 min e analisado (Silva, 1999). Os resultados da análise foram: $\mathrm{pH}$ em $\mathrm{CaCl}_{2}$ 0,001M, 5,9; matéria orgânica (MO), $15,4 \mathrm{~g} / \mathrm{kg}$; $\mathrm{P}$ resina, $3,1 \mathrm{mg} / \mathrm{dm}^{3} ; \quad \mathrm{K}, \quad 0,75 \mathrm{mmol}_{\mathrm{c}} / \mathrm{dm}^{3}$; $\mathrm{Ca}, 19,08 \mathrm{mmol}_{\mathrm{C}} / \mathrm{dm}^{3} ; \mathrm{Mg}, 15,96 \mathrm{mmol}_{\mathrm{c}} / \mathrm{dm}^{3} ; \mathrm{Na}$, $3,65 \mathrm{mmol}_{\mathrm{c}} / \mathrm{dm}^{3} ; \mathrm{Cu}, 0,48 \mathrm{mg} / \mathrm{dm}^{3} ; \mathrm{Fe}, 9,36 \mathrm{mg} / \mathrm{dm}^{3} ; \mathrm{Mn}$, $7,57 \mathrm{mg} / \mathrm{dm}^{3} ; \mathrm{Zn}, 2,67 \mathrm{mg} / \mathrm{dm}^{3} ; \mathrm{SB}, 39,44 \mathrm{mmol}_{\mathrm{c}} / \mathrm{dm}^{3}$; CTC, $48,44 \mathrm{mmol}_{\mathrm{c}} / \mathrm{dm}^{3} \mathrm{e} \mathrm{V}, 81,42 \%$.

Amostras de 1,5 L do solo autoclavado foram colocadas em vasos de plástico e umedecidas pela ativação intermitente de um sistema de nebulização instalado no interior da casa de vegetação. A ativação desse sistema ocorreu três a quatro vezes, durante 15 minutos, nos dias ensolarados. Após o umedecimento inicial, aplicaram-se, na camada superficial, os inóculos: $40 \mathrm{~g}$ da mistura CA1, $44 \mathrm{~g}$ da mistura $\mathrm{CA} 2,45 \mathrm{~g}$ do produto comercial e $45 \mathrm{~g}$ de solo e areia esterilizados.

A densidade de esporos de FMA dos inóculos foi estimada pela técnica do peneiramento úmido e da centrifugação em solução de sacarose (Sieverding, 1991). A massa das misturas CA1 e CA2 forneceu pelo menos 200 esporos de FMA por vaso, e do produto comercial em torno de 100 esporos de Glomus. Após a aplicação desses inóculos, distribuíram-se duas castanhas de caju por vaso, à profundidade de 2 a $3 \mathrm{~cm}$ no solo, com o ponto de incisão castanha/pedúnculo voltado para cima.

Após 15 dias da semeadura, correspondendo a dois e três dias após a germinação, procedeu-se o desbaste, deixando-se apenas uma planta por vaso, e aplicaramse as doses de fósforo. Aplicaram-se em metade dos vasos $50 \mathrm{~mL}$ de uma solução contendo $576 \mathrm{mg}$ de $\mathrm{K}_{2} \mathrm{HPO}_{4}$ p.a. (131 mg de $\mathrm{P}$, equivalente a $87 \mathrm{mg}$ de $\mathrm{P}$ por litro de solo). No tratamento sem $\mathrm{P}$, os vasos rece- beram $50 \mathrm{~mL}$ de uma solução contendo $314 \mathrm{mg}$ de $\mathrm{KCl}$, sendo a mesma quantidade de $\mathrm{K}$ fornecida com a adubação fosfatada. Cada planta recebeu mensalmente $50 \mathrm{~mL}$ da solução nutritiva Hoagland, sem fósforo (Sarruge, 1975).

Após dois e quatro meses da semeadura, as plantas foram avaliadas pelo crescimento em altura e diâmetro do caule. Ao final do segundo período, as plantas foram colhidas, separando-se do solo as raízes e destas a parte aérea. Das raízes foram separadas frações de aproximadamente $1 \mathrm{~g}$, para avaliar a intensidade de colonização dos FMA. Essas porções de raízes finas (em torno de $1 \mathrm{~mm}$ de diâmetro) foram conservadas em solução alcoólica (50\% de etanol) até iniciar o processo de descoramento. A coloração das raízes foi realizada conforme método de Phillips \& Hayman (1970) modificado. A modificação consistiu em descoramento das raízes com solução alcalina ( $\mathrm{KOH}$ a $10 \%)$ em autoclave, a $121^{\circ} \mathrm{C}$ durante $12 \mathrm{~min}$; lavagem em água várias vezes; cobertura com água oxigenada diluída $\left(\mathrm{H}_{2} \mathrm{O}_{2}\right.$ a $\left.1,25 \%\right)$, durante $20 \mathrm{~min}$; acidificação com solução a $1 \%$ de $\mathrm{HCl}$, durante $5 \mathrm{~min}$; coloração com azul de anilina em solução lacto-glicérica $(875 \mathrm{~mL}$ de ácido lático, $63 \mathrm{~mL}$ de glicerina, $0,5 \mathrm{~g}$ do corante e $62 \mathrm{~mL}$ de água destilada) durante a noite; lavagem em água. Foram avaliados 20 segmentos de aproximadamente $1 \mathrm{~cm}$ de cada planta, montados em lâminas de vidro e cobertos com solução lacto-glicérica e lamínulas, para observar ao microscópio (100 a 400 vezes) a presença de micélio, vesículas e outras estruturas dos fungos no interior das raízes. A porcentagem de colonização foi determinada com base na colonização intra-radicular dos FMA. A fração restante das raízes foi observada sob lupa, para avaliar o micélio fúngico externo às raízes.

As frações restantes das raízes e a parte aérea das plantas foram secadas em estufa de circulação forçada de ar até obter massa constante. Na parte aérea seca e triturada foram determinados os teores de macronutrientes e micronutrientes (Silva, 1999).

Os dados de crescimento foram utilizados no cálculo do incremento proporcionado pelos FMA, utilizando a fórmula $100[(\mathrm{X}-\mathrm{Y}) / \mathrm{Y}]$, em que $\mathrm{X}$ representou a característica da planta micorrizada e $\mathrm{Y}$ a da planta controle. A eficiência dos FMA foi calculada com base na massa da matéria seca total das plantas pela fórmula $100[(\mathrm{X}-\mathrm{Y}) / \mathrm{X}]$.

Os dados de crescimento, colonização radicular e acúmulo de nutrientes foram submetidos à análise de variância, com nível de significância de 5\% pelo teste $\mathrm{F}$, e as médias comparadas pelo teste de Tukey a $5 \%$ de 
probabilidade. Os valores das características de crescimento e o conteúdo de nutrientes da parte aérea das plantas foram transformados em $\mathrm{x}^{0,5} \mathrm{e}$ a colonização micorrízica, em arco seno $(\mathrm{x} / 100)^{0,5}$, antes de proceder a análise estatística.

\section{Resultados e Discussão}

$\mathrm{O}$ crescimento das mudas do cajueiro-anão-precoce CCP 76 foi influenciado pela inoculação de fungos MA e pela adubação, mas não houve interação entre esses fatores (Tabela 1). Aos dois meses da semeadura, observou-se incremento no diâmetro do caule das plantas que receberam adubação fosfatada $(87 \mathrm{mg} / \mathrm{L}$ de $\mathrm{P})$ e não houve resposta à inoculação de fungos MA.

A resposta à adubação fosfatada durante a fase inicial de crescimento das plantas indica que o $\mathrm{P}$ disponível no solo $\left(3,1 \mathrm{mg} / \mathrm{dm}^{3}\right)$ e proveniente das reservas dos cotilédones era insuficiente. A massa da matéria fresca das castanhas utilizadas era de aproximadamente $8 \mathrm{~g}$ cada. Já a ausência de resposta à inoculação dos FMA, durante os dois meses iniciais, pode ser decorrente dos nutrientes de reserva e da baixa colonização micorrízica das mudas. Haugen \& Smith (1993), aplicando a mistura NutriLink, contendo Glomus intraradices, no viveiro, e removendo os cotilédones das plântulas do cajueiro recém-germinadas, observaram maior taxa de colonização micorrízica arbuscular $(55 \%)$, em relação às plantas que permaneceram com cotilédones. Porém, sua remoção nas plântulas não resultou em maior vigor dessas mudas. Ademais, Cardoso (1994) observou ausência de resposta do cajueiro CCP 06, aos 60 dias de idade, quando aplicou suspensões com 50 esporos das espécies Glomus sinuosum, Glomus macrocarpum, Glomus versiforme, Glomus sp., Scutelospora heterogama e Scutelospora sp., em separado, nas raízes das plântulas recém-germinadas.

Aos quatro meses da semeadura, observou-se melhor desempenho das mudas micorrizadas (Tabela 1). Houve incremento no diâmetro do caule, na produção de massa de matéria seca das plantas colonizadas por fungos MA exóticos da mistura Mycogold, em relação às plantas controle. A aplicação da mistura CA1, composta de Glomus etunicatum, G. glomerulatum (espécies com maior número de esporos), Scutellospora sp. e Acaulospora foveata (espécies com menor número de esporos no inóculo) também proporcionou maior crescimento em diâmetro do caule e maior acúmulo de massa de matéria seca na parte aérea das plantas. Por sua vez, o tratamento com a mistura CA2 contendo G. etunicatum, Entrophospora sp. e Scutellospora sp. foi significativamente inferior ao tratamento com a mistura CA1 para o diâmetro do caule e não resultou em diferenças significativas na produção de matéria seca da raiz e da parte aérea, em relação aos demais tratamentos. Isto indica que as comunidades de FMA nativos promovem respostas diferenciadas na promoção do crescimento das plantas.

Tabela 1. Altura e diâmetro do caule, aos 60 dias da semeadura, crescimento aéreo, acúmulo de matéria seca das raízes e da parte aérea e colonização micorrízica, aos 120 dias da semeadura do cajueiro-anão-precoce CCP 76, em razão da inoculação de FMA nativos das misturas CA1 (Glomus etunicatum, G. glomerulatum, Scutellospora sp. e Acaulospora foveata) e CA2 (G. etunicatum, Entrophospora sp. e Scutellospora sp.) e de fungos exóticos da mistura comercial Mycogold e da aplicação de fósforo no solo ${ }^{(1)}$.

\begin{tabular}{|c|c|c|c|c|c|c|c|c|c|c|c|c|c|}
\hline \multirow[t]{3}{*}{ Tratamento } & \multicolumn{4}{|c|}{60 dias } & \multicolumn{9}{|c|}{120 dias } \\
\hline & \multicolumn{2}{|c|}{ Altura } & \multicolumn{2}{|c|}{ Diâmetro } & \multicolumn{2}{|c|}{ Altura } & \multicolumn{2}{|c|}{ Diâmetro } & \multicolumn{2}{|c|}{ Massa da raiz } & \multicolumn{2}{|c|}{$\begin{array}{l}\text { Massa da } \\
\text { parte aérea }\end{array}$} & \multirow[t]{2}{*}{$\begin{array}{l}\text { Colonização } \\
\text { radicular (\%) }\end{array}$} \\
\hline & $\mathrm{cm}$ & $\mathrm{I}^{(2)}$ & $\mathrm{mm}$ & I & $\mathrm{cm}$ & I & $\mathrm{mm}$ & I & $\mathrm{g}$ & I & $\mathrm{g}$ & I & \\
\hline \multicolumn{14}{|c|}{ Inóculo fúngico } \\
\hline CA1 & $34,2 \mathrm{a}$ & 6,2 & $7,3 \mathrm{a}$ & 1,4 & $45,6 \mathrm{a}$ & 10,9 & $12,4 \mathrm{a}$ & 10,7 & $3,44 \mathrm{ab}$ & 21,1 & $11,06 \mathrm{a}$ & 17,1 & $87,1 \mathrm{a}$ \\
\hline $\mathrm{CA} 2$ & $34,4 \mathrm{a}$ & 6,8 & $7,3 \mathrm{a}$ & 1,4 & $44,0 \mathrm{a}$ & 7,1 & $11,6 \mathrm{bc}$ & 3,6 & $3,42 \mathrm{ab}$ & 20,4 & $10,88 \mathrm{ab}$ & 16,4 & $79,0 \mathrm{a}$ \\
\hline Mycogold & $33,8 \mathrm{a}$ & 5,0 & $7,3 \mathrm{a}$ & 1,4 & $45,4 \mathrm{a}$ & 10,5 & $12,1 \mathrm{ab}$ & 8,0 & $4,01 \mathrm{a}$ & 41,2 & $11,05 \mathrm{a}$ & 14,6 & $75,1 \mathrm{a}$ \\
\hline Controle & $32,2 \mathrm{a}$ & - & $7,2 \mathrm{a}$ & - & $41,1 \mathrm{a}$ & - & $11,2 \mathrm{c}$ & - & $2,84 b$ & & $9,16 b$ & - & $0,0 \mathrm{~b}$ \\
\hline \multicolumn{14}{|c|}{$\mathrm{P}(\mathrm{mg} / \mathrm{L}$ de solo $)$} \\
\hline 0 & $33,7 \mathrm{a}$ & & $7,0 \mathrm{~b}$ & & $44,1 \mathrm{a}$ & & $11,7 \mathrm{a}$ & & $3,27 \mathrm{a}$ & & $10,46 a$ & & $56,8 \mathrm{a}$ \\
\hline 87 & $33,5 \mathrm{a}$ & & $7,5 \mathrm{a}$ & & $44,0 \mathrm{a}$ & & $11,9 \mathrm{a}$ & & $3,58 \mathrm{a}$ & & $10,61 \mathrm{a}$ & & $53,3 \mathrm{a}$ \\
\hline$\overline{\mathrm{CV}(\%)}$ & 5,5 & & 3,7 & & 6,0 & & 3,4 & & 10,6 & & 7,8 & & 31,2 \\
\hline
\end{tabular}


A presença de vesículas e de micélio fúngico foi constatada na região do córtex das raízes de todas as plantas que receberam inóculos de FMA, e a intensidade de colonização foi estimada em $75,1 \%$ a $87,1 \%$. Essa elevada taxa de colonização micorrízica pode ser atribuída ao tipo de inóculo (solo, raízes, micélio e esporos de FMA), à densidade de propágulos nas misturas (entre outras estruturas infectivas foram 200 esporos de FMA nativos e 100 esporos fúngicos do produto comercial) e à forma de aplicação no solo (em camada) dos vasos. Sieverding (1991), Oliveira et al. (1992), Siqueira et al. (1994) e Saggin Junior \& Siqueira (1996) relataram a maior eficiência da micorrização pelo uso de inóculos constituídos da mistura de solo, raízes e propágulos fúngicos, em comparação com suspensões de esporos de fungos micorrízicos.

A eficiência ou capacidade dos FMA em beneficiar as plantas na produção de massa de matéria seca foi superior no tratamento sem fosfato, em que variou de $19 \%$ da mistura comercial (Mycogold) a 20,8\% no tratamento com CA2 (Tabela 2). Por sua vez, com adubação fosfatada, os fungos da mistura CA2 apresentaram a menor eficiência $(10,6 \%$ ), quando os fungos exóticos da mistura comercial foram mais eficientes $(15,3 \%)$ na produção de matéria seca das plantas. Esses resultados indicam que FMA nativos poderiam estar mais adequados à condição de baixa fertilidade do solo, uma vez que foram isolados de raízes de cajueiros adultos, em pomares não adubados. Por sua vez, os FMA exóticos da mistura comercial, indicados para diversas espécies de plantas, podem ter sido selecionados numa condição de solo mais fértil. Infere-se que atributos do solo também devem ser levados em conta na seleção de FMA efetivos na cultura. Saggin Junior \& Siqueira (1996) relataram alta frequiência de ocorrência de espécies do gêne-

Tabela 2. Eficiência de FMA nativos das misturas CA1 (Glomus etunicatum, G. glomerulatum, Scutellospora sp. e Acaulospora foveata) e CA2 (G. etunicatum, Entrophospora sp. e Scutellospora sp.) e de fungos exóticos da mistura comercial Mycogold no acúmulo de matéria seca total (g/planta) das mudas do cajueiro-anão-precoce $\mathrm{CCP} 76$, em razão da aplicação ou não de fósforo no solo, aos 120 dias da semea$\operatorname{dura}^{(1)}$.

\begin{tabular}{ccccc}
\hline $\mathrm{P}(\mathrm{mg} / \mathrm{L}$ de solo) & Fungo CA1 & Fungo CA2 & Mycogold & Média \\
\hline 0 & 19,3 & 20,8 & 19,0 & 19,7 \\
87 & 15,0 & 10,6 & 15,3 & 13,6 \\
\hline
\end{tabular}

${ }^{(1)}$ Eficiência $=100[(\mathrm{X}-\mathrm{Y}) / \mathrm{X}]$, sendo $\mathrm{X}$ a característica da planta micorrizada e $\mathrm{Y}$ a característica da planta não micorrizada. ro Acaulospora no sistema radicular do cafeeiro da região Sudeste do país, mas detectaram maiores benefícios nas plantas com inoculação das espécies dos gêneros Gigaspora e Glomus.

A eficiência de FMA neste trabalho não foi muito expressiva quando comparada com os benefícios obtidos em outras fruteiras (Tabela 2). Isto pode sugerir que a muda do cajueiro-anão-precoce $\mathrm{CCP} 76$ é pouco dependente da associação micorrízica, e, considerando trabalhos com FMA (Haugen \& Smith, 1993; Cardoso, 1994) e adubação de mudas do cajueiro (Oliveira et al., 2000; Lima et al., 2001), essa resposta não deve mudar muito em outros clones. Maior benefício da micorrização foi observado em porta-enxertos de citros (Oliveira et al., 1992) e variedades de mamoeiro (Trindade et al., 2001). Em revisão sobre FMA em fruteiras, Siqueira \& Franco (1988) relataram um incremento relativo próximo a $200 \%$ em relação ao cajueiro, mais de $600 \%$ para o mamoeiro e mais de $1.200 \%$ para os citros.

Observou-se variação no conteúdo de alguns nutrientes da parte aérea em razão dos inóculos fúngicos e aumento no acúmulo de $\mathrm{P}$, com a adubação fosfatada, aos quatros meses da semeadura (Tabela 3 ). A inoculação de FMA do produto comercial propiciou maior acúmulo de $\mathrm{N}, \mathrm{P}$ e $\mathrm{K}$, ao passo que a mistura CA2 favoreceu a absorção de $\mathrm{Ca}$ e $\mathrm{Mg}$, em relação às plantas controle. Ademais, houve maior acumulação de Fe na parte aérea das plantas micorrizadas, que não diferiram entre si na absorção de outros nutrientes. Estes resultados estão condizentes com as observações feitas em relação ao crescimento das mudas (Tabela 1), permitindo sugerir que também podem ser obtidos benefícios com a micorrização das mudas do cajueiro.

A falta de resposta ao $\mathrm{P}$, aos quatro meses da semeadura, pode estar relacionada a um desequilíbrio na disponibilidade de outros elementos no solo, uma vez que o crescimento e a massa de matéria seca das plantas não foram influenciados pelo $\mathrm{P}$ aplicado (Tabela 1). Além disso, a baixa dependência do cajueiro ao $\mathrm{P}$ pode estar relacionada com a baixa eficiência de FMA (Tabela 2). Cardoso (1994) não constatou resposta do cajueiro CCP 06 ao P, aos 60 dias de idade. Lima et al. (2001) observaram também ausência de resposta do clone CCP 76, aos 75 dias de idade, quando aplicaram fertilizantes minerais $(1,28 \mathrm{~g}$ de uréia, $0,8 \mathrm{~g}$ de superfosfato triplo e $0,8 \mathrm{~g}$ cloreto de potássio) em sacolas de plástico com $2,5 \mathrm{~kg}$ de solo. Oliveira et al. (2000) recomendaram aplicar no cajueiro $50 \mathrm{~g}$ de $\mathrm{P}_{2} \mathrm{O}_{5}$ e $40 \mathrm{~g}$ de $\mathrm{K}_{2} \mathrm{O}$ por planta por ocasião da formação do pomar. 
Tabela 3. Conteúdo de nutrientes da parte aérea das mudas do cajueiro CCP 76, aos quatro meses da semeadura, em razão da inoculação de FMA nativos das misturas CA1 (Glomus etunicatum, G. glomerulatum, Scutellospora sp. e Acaulospora foveata) e CA2 (G. etunicatum, Entrophospora sp. e Scutellospora sp.) e de fungos exóticos da mistura comercial Mycogold e da aplicação de fósforo no solo ${ }^{(1)}$.

\begin{tabular}{|c|c|c|c|c|c|c|c|c|c|c|c|}
\hline Tratamento & $\mathrm{N}$ & $\mathrm{P}$ & K & $\mathrm{Ca}$ & $\mathrm{Mg}$ & S & $\mathrm{Na}$ & $\mathrm{Cu}$ & $\mathrm{Fe}$ & $\mathrm{Mn}$ & $\mathrm{Zn}$ \\
\hline & & & & pl & & & & & & & \\
\hline \multicolumn{12}{|c|}{ Inóculo fúngico } \\
\hline CA1 & $192,05 \mathrm{ab}$ & $12,86 \mathrm{ab}$ & $535,54 \mathrm{ab}$ & $25,38 \mathrm{ab}$ & $29,28 \mathrm{ab}$ & $22,44 a$ & $372,22 \mathrm{a}$ & $106,73 a$ & $2.003,46 a$ & $665,31 \mathrm{a}$ & $817,05 a$ \\
\hline CA2 & $192,57 \mathrm{ab}$ & $12,55 \mathrm{ab}$ & $593,41 \mathrm{ab}$ & $31,13 \mathrm{a}$ & $34,54 \mathrm{a}$ & $21,79 \mathrm{a}$ & $349,74 a$ & $87,29 \mathrm{a}$ & $1.948,27 \mathrm{a}$ & $751,18 \mathrm{a}$ & $816,58 a$ \\
\hline Mycogold & $202,71 \mathrm{a}$ & $13,04 \mathrm{a}$ & $596,16 \mathrm{a}$ & $30,34 \mathrm{ab}$ & $30,03 \mathrm{ab}$ & $21,65 \mathrm{a}$ & $412,66 a$ & $90,88 \mathrm{a}$ & $1.975,40 \mathrm{a}$ & $766,49 a$ & $864,36 a$ \\
\hline Controle & $162,17 \mathrm{~b}$ & $9,89 \mathrm{~b}$ & $405,20 \mathrm{~b}$ & $20,72 b$ & $24,00 \mathrm{~b}$ & $19,05 \mathrm{a}$ & $379,99 \mathrm{a}$ & $72,86 a$ & $1.336,83 \mathrm{~b}$ & $661,66 \mathrm{a}$ & $745,65 \mathrm{a}$ \\
\hline \multicolumn{12}{|c|}{$\overline{\mathrm{P}(\mathrm{mg} / \mathrm{L} \text { de solo) }}$} \\
\hline 0 & $191,42 \mathrm{a}$ & $10,47 b$ & $536,88 \mathrm{a}$ & $28,41 \mathrm{a}$ & $28,26 \mathrm{a}$ & $22,06 \mathrm{a}$ & $384,56 a$ & $86,66 a$ & $1.890,58 \mathrm{a}$ & $722,06 a$ & $845,05 a$ \\
\hline 87 & $183,56 a$ & $13,71 \mathrm{a}$ & $528,27 \mathrm{a}$ & $29,37 \mathrm{a}$ & $30,67 \mathrm{a}$ & $20,41 \mathrm{a}$ & $372,24 \mathrm{a}$ & $92,22 \mathrm{a}$ & $1.744,39 \mathrm{a}$ & $698,26 a$ & $777,06 \mathrm{a}$ \\
\hline $\mathrm{CV}(\%)$ & 6,5 & 8,5 & 10,4 & 10,6 & 10,5 & 7,0 & 8,6 & 12,9 & 7,9 & 14,6 & 7,6 \\
\hline
\end{tabular}

${ }^{(1)}$ Médias seguidas da mesma letra não diferem entre si pelo teste de Tukey a $5 \%$ de probabilidade; os valores foram transformados em $\mathrm{x}^{0,5}$ para a análise.

\section{Conclusões}

1. O cajueiro-anão-precoce $\mathrm{CCP} 76$, fase de muda, apresenta pequena resposta ao fósforo aplicado no solo.

2. A associação simbiótica com fungos micorrízicos arbusculares nativos (mistura de Glomus etunicatum, G. glomerulatum, Scutellospora sp. e Acaulospora foveata) e exóticos (mistura comercial Mycogold) é vantajosa para o desenvolvimento das mudas do cajueiro-anão-precoce CCP 76.

\section{Referências}

AGRIANUAL: anuário da agricultura brasileira. São Paulo: FNP Consultoria, 2002. 536p.

ALMEIDA, F.A.G.; MARTINS JUNIOR, W.; ALMEIDA, F.C.G. Fenologia comparativa de dois clones enxertados de cajueiro anão em condições de irrigação. Ciência Rural, v.32, p.221-228, 2002.

BARROS, L.M.; CAVALCANTI, J.J.V.; PAIVA, J.R. de; CRISÓSTOMO, J.R.; CORRÊA, M.P.F.; LIMA, A.C. Seleção de clones de cajueiro-anão para o plantio comercial no Estado do Ceará. Pesquisa Agropecuária Brasileira, v.35, p.2197-2204, 2000.

CARDOSO, B. de B. Efeitos da inoculação de fungos micorrízicos arbusculares e da adubação mineral fosfatada sobre o crescimento de porta-enxerto de cajueiro-anão-precoce (Anacardium occidentale L.). 1994. 46p. Dissertação (Mestrado) Universidade Federal do Ceará, Fortaleza.

CHAVES, J.C.M.; CAVALCANTI JUNIOR, A.T.; CORREIA, D.; SOUZA, F.X. de; ARAÚJO, C.A.T. Normas de produção de mudas. Fortaleza: Embrapa-CNPAT, 2000. 37p. (Documentos, 41).

CHULAN, A.; YACOB, O.; KAMAL, A.J.M.; PARANANTHAN, S. Distribution of Va mycorrhizal spores in sandy beach soils under cashew. Pertanika, v.6, p.15-20, 1983.
GHINI, R. Desinfestação do solo com uso de energias solar: solarização e coletor solar. Jaguariúna: Embrapa-CNPMA, 1997. 29p. (Circular, 1).

HAUGEN, L.M.; SMITH, S.E. The effect of inoculation of cashew with nutrilink on vesicular-arbuscular mycorrhizal infection and plantgrowth. Australian Journal of Agricultural Research, v.44, p.1211-1220, 1993.

JOHN, T.S. Uma lista de espécies de plantas tropicais brasileiras naturalmente infectadas com micorriza vesicular-arbuscular. Acta Amazonica, v.10, p.229-234, 1980.

LIMA, R. de L.S. de; FERNANDES, V.L.B.; OLIVEIRA, V.H. de; HERNANDEZ, F.F.F. Crescimento de mudas de cajueiro-anãoprecoce 'CCP76' submetidas a adubação orgânica e mineral. Revista Brasileira de Fruticultura, v.23, p.391-395, 2001.

OLIVEIRA, A.A.R.; WEBER, O.B.; SILVA, A.C.G.M. da. Micorrização e crescimento de porta-enxertos de citros sem função de inóculos micorrízicos vesículo-arbusculares. Pesquisa Agropecuária Brasileira, v.27, p.1049-1056, 1992.

OLIVEIRA, F.N.S.; AQUINO, A.R.L.; LIMA, A.A.C. Correção da acidez e adubação mineral em solos de cerrados cultivados com cajueiro-anão-precoce enxertado. Fortaleza: EmbrapaCNPAT, 2000. 32p. (Circular Técnica, 5).

OLIVEIRA, F.N.S.; RAMOS, A.D. Uso e manejo do solo. In: ARAÚJO, J.P.P. de; SILVA, V.V. da (Org.). Cajucultura: modernas técnicas de produção. Fortaleza: Embrapa-CNPAT, 1995. p.149150

OLIVEIRA, V.H.; BARROS, L.M.; LIMA, R.N. de. Influência da irrigação e do genótipo na produção de castanha em cajueiro anão precoce. Pesquisa Agropecuária Brasileira, v.38, p.61-66, 2003.

PHILLIPS, J.M.; HAYMAN, D.S. Improved procedures for clearing roots and staining parasitic and vesicular arbuscular-mycorrhizal fungi for rapid assessment of infection. Transactions of the British Mycological Society, v.55, p.158-161, 1970.

SAGGIN JÚNIOR, O.J.; SIQUEIRA, J.O. Micorrizas arbusculares em cafeeiro. In: SIQUEIRA, J.O. (Ed.). Avanços em fundamentos e aplicação de micorrizas. Lavras: UFL, 1996. p.203-254. 
SARRUGE, J.R. Soluções nutritivas. Summa Phytopathologica, v.1, p.231-233, 1975.

SHENCK, N.C.; PEREZ, Y. Manual for the identification of VA mycorrhizal fungi. $2^{\text {nd }}$ ed. Gainsville: International Culture Collection of VA Mycorrhizal Fungi; University of Florida, 1988. $241 \mathrm{p}$.

SIEVERDING, E. Vesicular-arbuscular mycorrhiza management in tropical agrosystems. Eschborn: Deutsche Gesellschaft für Technische Zusammenarbeit, 1991. 371p.

SILVA, F.C. (Org.). Manual de análises químicas de solos, plantas, e fertilizantes. Brasília: Embrapa SCT; Embrapa CNPS; Embrapa CNPTIA, 1999. 370p.
SIQUEIRA, J.O.; FRANCO, A.A. Biotecnologia do solo: fundamentos e perspectivas. Brasília: MEC; ABEAS, 1988. 235p.

SIQUEIRA, J.O.; COLOZZI FILHO, A.; SAGGIN JÚNIOR, O.J. Efeitos da infecção de plantas de cafeeiro com quantidades crescentes de esporos do fungo Gigaspora margarita. Pesquisa Agropecuária Brasileira, v.29, p.875-883, 1994.

SOUZA, F.X. de. Materiais para formulação de substratos na produção de mudas e no cultivo de plantas envasadas. Fortaleza: Embrapa-CNPAT, 2001. 21p. (Docummentos, 43).

TRINDADE, A.V.; SIQUEIRA, J.O.; ALMEIDA, F.P. de. Dependência micorrízica de variedades comerciais de mamoeiro. Pesquisa Agropecuária Brasileira, v.36, p.1485-1494, 2001.

$\overline{\text { Recebido em } 17 \text { de setembro de } 2003 \text { e aprovado em } 20 \text { de fevereiro de } 2004}$ 